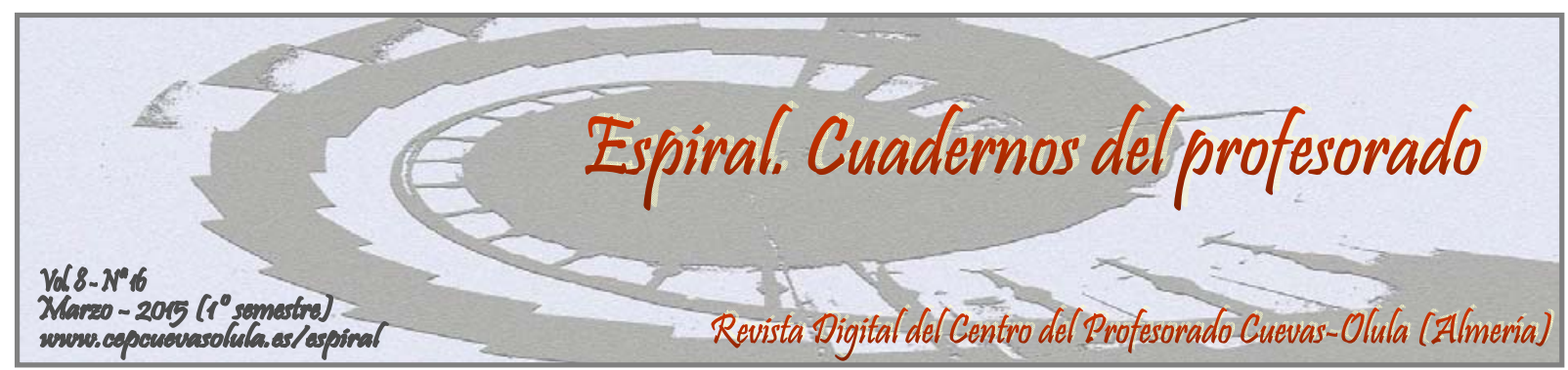

\title{
CORREMONTES. CORRER POR MONTAÑA CON ESCOLARES
}

\section{MOUNTAINRUNNERS. TRAIL RUNNING FOR CHILDREN}

\section{Darío Pérez-Brunicardi ${ }^{(1)}$ y María Teresa Archilla Prat ${ }^{(2)}$}

(1) Facultad de Educación de Segovia, Universidad de Valladolid, España

(2) Profesora de Educación Secundaria, Comunidad de Madrid, España

\begin{abstract}
RESUMEN: Durante los últimos años se han extendido de manera significativa diferentes iniciativas de aprendizaje de la carrera en Educación Física, tanto en Educación Primaria como en Secundaria, basadas en la autonomía y el mantenimiento del ritmo constante que les permita realizar esfuerzos de larga duración. Simultáneamente, se está produciendo una gran evolución de las carreras por montaña, tanto en su dimensión de rendimiento como en la popular. Transfiriendo los trabajos del contexto de Educación Física a la promoción de las carreras por montaña entre escolares hemos desarrollado varios proyectos con resultados muy interesantes. Este documento muestra, de manera resumida, las bases conceptuales y las estrategias prácticas empleadas para el aprendizaje de la carrera de larga duración por montaña. Su finalidad es impulsar la carrera por montaña desde un enfoque participativo en los contextos escolar y no escolar, superando el estereotipo de que la carrera es necesariamente sinónimo de rendimiento. Así mismo, se presentan diferentes modos de organizar carreras por montaña con escolares sin necesidad de clasificaciones, cuya motivación principal sea el placer de correr por la naturaleza.
\end{abstract}

Palabras clave: correr por montaña, carrera de larga duración, Educación Física, aprendizaje comprensivo.

ABSTRACT: During recent years, lot of experiences is developed about running in Physical Education on Primary and Secondary Spanish Schools. These proposals are focused on promoting the habit of running longer. At the same time, trail-running races are becoming more popular, surpassing myth that these races are only for supermen. We have developed different experiences transferring methods of learning long-time running to learning trail-running. The aims of this model are to promote the love for running in nature on healthy way. This paper shows the theoretical foundations of this learning model and practical proposals to develop training sessions and to manage non-performance races for children.

Key words: trail running, long time running, Physical Education, comprehensive learning.

Pérez-Brunicardi, D. y Archilla Prat, M. T. (2015). Corremontes. Correr por montaña con escolares. Espiral. Cuadernos del Profesorado, 8(16), 74-83. Disponible en: http://www.cepcuevasolula.es/espiral.

Fecha de recepción: 27/07/2014

Fecha de aceptación: 04/011/2014
Enviar correspondencia a: dariopb@mpc.uva.es 


\section{1.- A LOS NIÑOS Y NIÑAS LES GUSTA CORRER}

Generalmente a los niños y niñas les gusta correr. La mayoría de sus juegos espontáneos, si se les permite, se basan en carreras, sólo coartadas por las limitaciones espaciales, los hábitos sedentarios o la "aversión al riesgo" (Gill, 2007, 2010) de los adultos responsables. La mayoría de estos juegos son de persecución, desarrollando mediante el juego la función innata y atávica del cazador y la presa. Sin embargo, cuando este doble objetivo lúdico se diluye o se pierde completamente, es difícil mantener la motivación por correr. El deporte viene a ser el sustituto de este juego infantil, incorporando las marcas y las clasificaciones como elemento motivador. Como podemos apreciar, existe una diferencia significativa entre la motivación de uno y otro tipo de carrera: mientras que en el juego la motivación es intrínseca, en el deporte la motivación por correr es extrínseca.

¿Qué pasa cuando esa motivación extrínseca desaparece? El deporte, en su formato convencional, establece una regla tácita que parece inquebrantable: sólo puede haber un ganador. Por tanto, el resto no lo son, son perdedores. No todos alcanzarán el éxito. Si éste es el único o principal elemento motivador, el desánimo y el abandono es cuestión de tiempo. Sin embargo, para evitarlo, tradicionalmente se han buscado alternativas. Por un lado, esta regla se suele mitigar con "la clasificación”. Su mínima expresión es el pódium, en el que algunas personas más disfrutarán de la gloria del éxito, aunque en diferentes niveles de reconocimiento (altura del cajón, tamaño del trofeo, material de la medalla, cantidad o valor de los premios, etc.). En edad infantil, éste recurso es, a todas luces, insuficiente para mantener la motivación de todos y todas de forma permanente. Cuando vayan descubriendo que nunca subirán a uno de esos cajones, el abandono es cuestión de tiempo. Por ello, a esta medida le siguen las tablas de clasificación, en las que cada uno se sitúa en un rango, según sus resultados (sus tiempos) y en la que el éxito puede ser relativo. Pueden multiplicarse los vencedores por el número de corredores que logran su reto personal (mejorar el tiempo, superar a determinado oponente, mejorar su posición en el pódium, etc.). Esta estrategia está teniendo muy buenos resultados entre los adultos, logrando que las carreras populares se conviertan en todo un fenómeno social en los últimos años. Si el objetivo no es sólo lograr campeones, sino generar adherencia y hábitos activos, no es suficiente, porque no es transferible a los escolares. Sus intereses y su visión de futuro no es la de los adultos. Niños y adolescentes necesitan una recompensa inmediata a su esfuerzo, a ser posible intrínseca. Las carreras, siguiendo el modelo convencional, sólo ofrecen esta recompensa a algunos. Con el tiempo, los que no obtienen la clasificación deseada se van retirando.

Para lograr esa recompensa intrínseca tenemos el difícil reto de conservar su motivación y su gusto por correr, como si fuera un valioso tesoro. Existen innumerables variables que pueden desequilibrar la balanza en cualquiera de los dos sentidos. Los que consideramos más decisivos son: a) la percepción positiva de la carrera de larga duración (CLD), b) la socialización en un ambiente confortable, c) la promoción de hábitos saludables y d) la elección de un entorno de práctica estimulante.

\section{EVITANDO PERCEPCIONES NEGATIVAS}

Con demasiada frecuencia, la carrera se ha empleado de un modo negligente en el contexto escolar y en el deporte base. Sus peores enemigos son:

- Los castigos en forma de carrera: dar vueltas al campo, por ejemplo. Algo que se ve con toda naturalidad y que supone para muchos la primera impronta negativa de la carrera en la infancia: esfuerzo fatigoso, impuesto por castigo y que priva del juego.

- La carrera continua en calentamientos no significativos, incomprensibles, aburridos, que en raras ocasiones los jóvenes entienden, impacientes en su deseo por comenzar a jugar.

- Los test de condición física, verdaderas torturas para los que no alcanzaban los mínimos y un elemento de presión cuando se empleaban como medio de calificación en la escuela o de selección en el deporte de competición.

- La presión de los adultos, entrenadores y familiares, para que el niño o la niña corran deprisa, adelanten, ganen. No se conforman: "hay que darlo todo", "podrías haberlo hecho 
mejor”, “¿cómo te ha ganado ese niño?”... palabras envenenadas (Fernández-Balboa, 2004) que oímos con cierta frecuencia en torno a la línea de llegada.

Si se les da margen, si se les permite aprender a encontrar su ritmo, si se les ayuda a entender los porqués, si se les enseña a mejorar con su propia motivación como motor de impulsión, las cosas cambian radicalmente. Uno de los elementos fundamentales son las sensaciones positivas durante la carrera. El esfuerzo debe conllevar suficiente recompensa por sí mismo. Hasta que encuentre esa recompensa intrínseca, la carrera debe mantenerse en un ritmo "llevadero", soportable, que permita mantener la carrera durante el tiempo o la distancia que uno desee. Éste es el primer objetivo de la CLD (Generelo, Julián, y Zaragoza, 2009) en general y de la carrera por montaña en particular: alargar la duración y la distancia. Las sensaciones negativas de sobreesfuerzo, si aún no han adquirido la motivación por el logro de una meta, suelen desmotivar y generar rechazo entre los principiantes.

\section{SOCIALIZACIÓN CONFORTABLE}

La motivación por la práctica de ejercicio físico es muy compleja y diversa, explicándose desde muy diferentes enfoques (Roberts et al, 1981; Roberts y Treasure, 2003, 2012). Sin embargo, sobre la necesidad del niño y del adolescente por relacionarse con sus compañeros y amigos no hay ninguna duda. Generar un ambiente positivo y confortable se convierte en una prioridad para lograr la adherencia a la práctica de la CLD. La rivalidad y la competitividad no suelen ayudar a la creación de este ambiente entre compañeros, al menos no entre todos ellos, desechando a los que no cumplan las exigencias. Sin embargo, ambientes de cooperación y compañerismo, dejando las marcas en un segundo plano, ayudan a que todos se encuentren incluidos y relajados para ir buscando sus propios retos personales.

\section{UN REGALO PARA TODA LA VIDA: PROMOCIÓN DE HÁBITOS SALUDABLES}

La CLD puede verse como un deporte de rendimiento o como un modo de lograr una vida activa y saludable. Generalmente, la carrera se enfoca desde el primer enfoque, asociada generalmente al atletismo. No parece frecuente ver niños corriendo por el simple hecho de estar en forma o sentirse saludables, ¿no? Es más normal que lo hagan en el contexto de grupos de entrenamiento deportivo o en clases de educación física escolar. Como hemos comentado, el niño corre de manera espontánea cuando está jugando. Sólo a través de una actividad dirigida, como el entrenamiento o la educación física, correrá por otros fines. Sin embargo, si desde pequeño sólo le enseñan a correr por deporte, la utilidad de la carrera para la salud sólo la descubrirá cuando sea adulto y sus metas sean otras. En ese momento, el adulto redescubre la carrera. ¿Cuántas personas conocemos que, sin haber practicado deporte nunca, encuentran un modo de vida en las carreras populares o en el "running”? No es necesario que el niño tarde tanto en descubrirlo.

La promoción de la salud hay que desarrollarla de manera experiencial, más allá del libro de texto o de las recomendaciones teóricas. Tampoco puede darse por sentada la idea de que el deporte educa para la salud por sí mismo. Es necesario que la CLD comience a formar parte de su vida desde el principio, con un enfoque orientado a la salud, además del deportivo o el lúdico. Esto es importante en aquellos niños, niñas y, especialmente, adolescentes que no se sienten motivados por la práctica deportiva. No encajan en sus exigencias, en sus clasificaciones, en la permanente necesidad de demostrar ser mejor. Los bien cualificados físicamente, cuya competencia percibida es positiva, tienen más fácil encontrar la motivación. Por tanto, aprender a quererse y a cuidarse, buscando unos hábitos de vida activos y saludables, pueden ser uno de los mejores anclajes para la adherencia a la práctica de la carrera durante toda la vida, donde correr más rápido no es tan importante como hacerlo durante más tiempo, tanto por la distancia recorrida como por los años vividos.

\section{ENTORNO DE PRÁCTICA POSITIVO Y ESTIMULANTE}

Es evidente que para lograr un ambiente positivo, el entorno de práctica juega un papel muy importante. Para que sea motivante, la variedad del entorno es una de las variables fundamentales para lograrlo. En instalaciones deportivas convencionales, la variedad es menor y sólo se puede lograr mediante la colocación de obstáculos artificiales. Esta puede ser una solución parcial. La naturaleza 
ofrece una "instalación” con una variedad sin límites. Desde los parques hasta los caminos de montaña, la naturaleza nos va a proporcionar diferentes superficies, pendientes, obstáculos, que van a enriquecer muchísimo el aprendizaje de la carrera, logrando que el niño sea más versátil motrizmente.

Pero la naturaleza ofrece mucho más que una buena pista de entrenamiento. La naturaleza penetra en el corredor por todos los sentidos, inundándole de sensaciones diferentes a las de la vida cotidiana. Las carreras por montaña, junto a la orientación, son el máximo exponente de la carrera por la naturaleza. No siempre es posible correr en terreno silvestre, pero que éste sea el objetivo aumenta mucho el interés de esta actividad. Así mismo, la naturaleza es un icono de salud. El ambiente limpio que nos ofrece reconforta tanto al cuerpo como a la mente y al espíritu, produciendo un efecto triplemente positivo en quien hace ejercicio físico en la naturaleza. Es cierto que la naturaleza también conlleva inconvenientes como la exposición a la intemperie y los rigores meteorológicos, a picaduras, rozaduras, contusiones; que es un entorno más inestable que una instalación deportiva homologada. Sin embargo, la incertidumbre, las sensaciones de aventura y el reto que ofrece el control del riesgo, son elementos altamente motivadores que convierten a la carrera en algo más que un deporte.

Cuando viajamos nos gustaría que el viaje no terminara nunca, descubriendo constantemente lugares nuevos. Del mismo modo, es esencial lograr aumentar la distancia y el tiempo de carrera si nos gusta recorrer la naturaleza, disfrutarla sin límites, y hacerlo sin fatiga, con sensaciones positivas, con la suficiente agilidad que nos permita salvar los obstáculos y disfrutar con ellos. Cuando el niño va descubriendo que evoluciona en este sentido, la recompensa es inmediata y la motivación aumenta. No es necesario competir con otros para sentir que ha logrado el reto. Por tanto, hay que buscar siempre la naturaleza, la mejor instalación deportiva. El resto serán siempre pobres imitaciones.

\section{APRENDER A CORRER POR MONTAÑA}

El origen de este modelo se encuentra en la enseñanza comprensiva de la CLD en la escuela, buscando el propio ritmo, la autonomía de los escolares y la motivación por aumentar la duración o la distancia. Lo descubrimos en las Jornadas de Educación Física de Huesca. Allí, Larraz (1998) nos expuso su trabajo en la escuela basado en la resistencia aeróbica. Fue la primera vez que lo que estábamos escuchando que no se basaba en los clásicos test de condición física. Inmediatamente, en el seno del Grupo de Trabajo Internivelar de Investigación - Acción en Educación Física de Segovia (Barba Martín y López Pastor, 2006; Barba Martín et al., 2007) nos pusimos a trabajar en buscar un modo de promover la carrera continua con autonomía en educación física, basándonos en la búsqueda del ritmo y en el aprendizaje comprensivo que permitiera entender los porqués que siempre se habían omitido. Simultáneamente se seguía trabajando en Huesca en este sentido, ofreciendo interesantes resultados (Generelo et al., 2009; Julián et al., 2012) o en los trabajos de Pérez-Pueyo (1999a y 1999b; 2010), entre otros.

El trabajo fue dando sus frutos en educación física escolar hasta que en 2010 se transfiere al desarrollo del programa de Deporte Escolar de Segovia, incorporándose en algunas sesiones del curso y en la versión escolar una carrera popular urbana (Figura 1). Esta carrera congregaba a los escolares de toda la ciudad en un modelo no competitivo, basado en mantener el ritmo que les permitiera los completar recorridos de diferentes longitudes por un parque de la ciudad. Esta actividad se ha normalizado en este programa como el principal modo de aprender a correr.

$\mathrm{Al}$ año siguiente (2011) este modelo de carrera infantil se transfiere y adapta en la Carrera Popular por Montaña "Vuelta a los

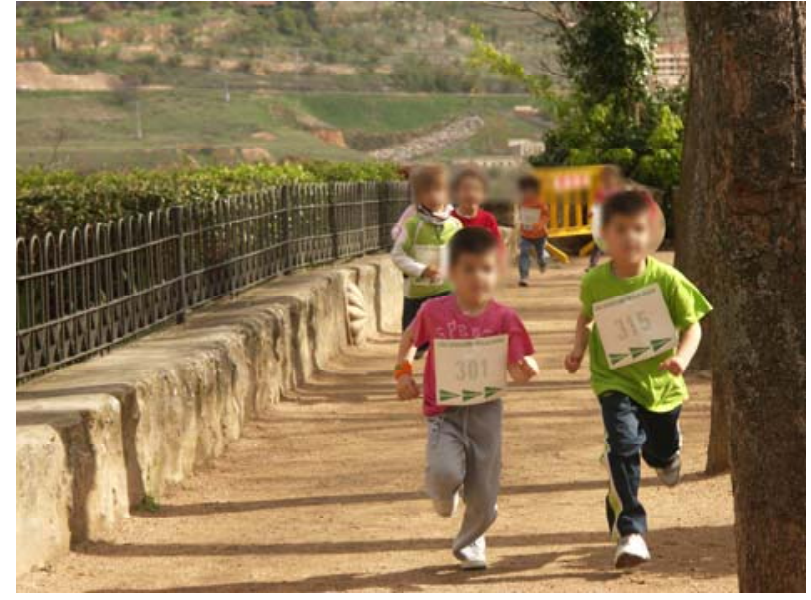

Figura 1. Corredores en la carrera del programa de Deporte Escolar de Segovia (2010). 
Jardines de La Granja” (Segovia), con diferentes recorridos que iban desde 500m a los $5 \mathrm{Km}$, adentrándose ya en terreno montañoso con poco desnivel (entre 50 y 100m). La experiencia positiva y la respuesta de los niños y niñas de todas las edades han mantenido esta prueba, rompiendo el tópico de que una carrera no tiene sentido si no se obtiene una clasificación y que los niños sólo corren y se esfuerzan motivados por la competición.

Durante el curso 2012/13 tenemos la oportunidad de desarrollar una experiencia con mayor continuidad, en el seno de la Escuela de Deportes en la Naturaleza de La Losa (Segovia). Este proyecto nos permitió centrar el programa en diferentes deportes, entre los que estaba la orientación deportiva, la carrera por montaña y el duatlón-cross (Figura 2). Todas ellas las desarrollamos basándonos en este modelo. Posteriormente participaron también en diferentes pruebas competitivas, con interesantes resultados en la clasificación y, mucho más importante, en su motivación hacia la actividad de aquellos

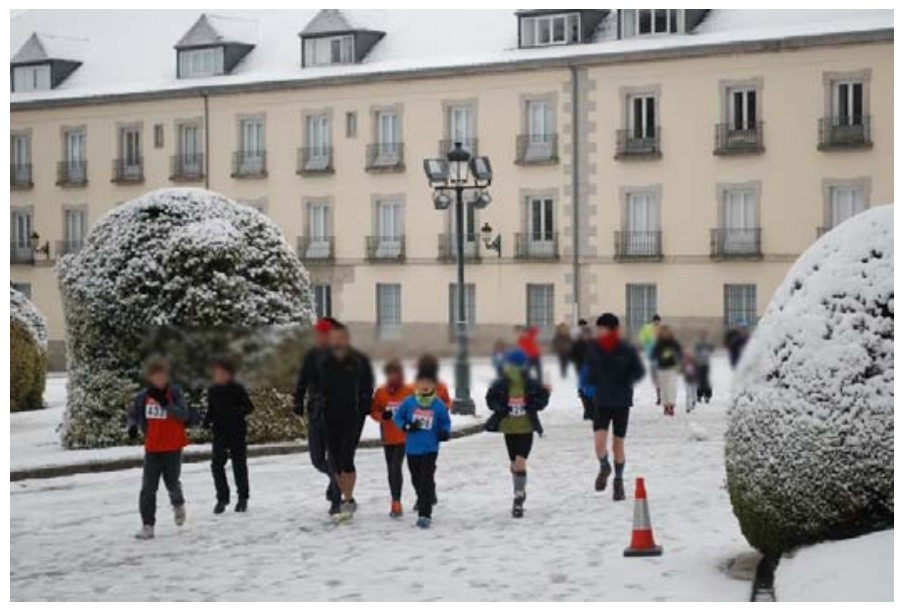

Figura 2. Grupo guiado en la Carrera Popular por Montaña Vuelta a los Jardines (2013).

que inicialmente saltaban hacia atrás cuando les decíamos que "tocaba correr”. Simultáneamente hicimos diferentes experiencias como una carrera en familia o un reto colectivo en un instituto, con resultados muy positivos de participación y motivación.

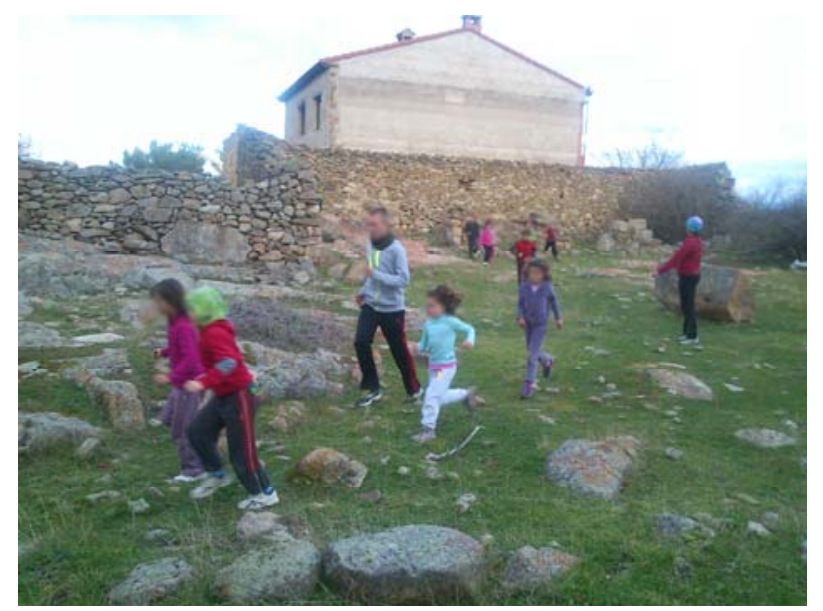

Figura 3. Entrenando por los alrededores del pueblo con la Escuela de Deportes en la Naturaleza (2013)
El último eslabón de esta cadena, en 2014, es el proyecto Corremontes, en colaboración con diferentes entidades (Grupo de montaña La Acebeda, Universidad de Valladolid, Federación de Deportes de Montaña, Escalada y Senderismo de Castilla y León y Areva Valsaín S.L.). Con el ánimo de promover la práctica de la carrera por montaña entre los más pequeños, sin necesidad de ligarlo a la competición, este proyecto intenta extender la aplicación de este modelo de aprendizaje de la CLD entre los clubes de montaña y escuelas de deportes en la naturaleza de Castilla y León. Asociado a este proyecto se han convocado un encuentro de jóvenes corredores en la Montaña Palentina y una jornada de formación de técnicos deportivos en León (Figura 3).

Algunas de las estrategias metodológicas de este modelo son las siguientes:

- Seleccionar recorridos amenos, divertidos, con obstáculos que estimulen el gusto por correr. Aunque este recorrido debe responder a las posibilidades de los participantes, lo interesante es que vaya por terrenos variados, con subidas y bajadas, rocas a las que poder subirse, obstáculos pequeños que saltar, veredas estrechas y zigzagueantes o amplias praderas para bajar “a tumba abierta”. Los recorridos pueden ser propuestos por el entrenador o profesor, aunque es interesante que ellos también vayan proponiendo los suyos propios. Esta estrategia va encaminada a aumentar la motivación, la variedad de recursos motrices y la versatilidad de la condición física. La carrera debe plantearse como un reto deseable y 
alcanzable, por lo que debe ser individualizado. Para ello, se puede recurrir a las siguientes estrategias:

a. Salir todo lo que se pueda al campo y a parques con recorridos divertidos, mostrando una actitud positiva, amena y paciente, lo que generará un ambiente lúdico y desenfadado que previene actitudes pasivas o negativas hacia el esfuerzo.

b. Desarrollar las actividades por niveles diferentes de práctica, promoviendo la inclusión de todos, independientemente de sus cualidades. Dejar que se establezcan de manera espontánea los grupos, en función de su ritmo de carrera. Si se detecta que esto puede generar un cierto grado de competitividad o rivalidad negativa, se pueden realizar salidas diferenciadas para que no se puedan establecer comparaciones inoportunas que desanimen a los más lentos.

c. Transferirles progresivamente autonomía en el desarrollo de las actividades y en la evaluación de sus resultados, lo que se va a reforzar una actitud responsable hacia el logro de sus objetivos.

- Cuando el terreno es más monótono (pistas deportivas o patios del colegio), donde generalmente se realizan los entrenamientos en circuito, conviene centrar la atención es aspectos comprensivos de la CLD. Los aspectos que necesitan entender son:

a. La relación entre la frecuencia cardiaca (FC) y el esfuerzo, empleando gráficas de pulso y tiempos en recorridos de longitud fija, que permitan mostrar cómo aumenta la frecuencia cardiaca cuando se realiza la vuelta en menor tiempo (Barba Martín et al., 2007; Generelo et al., 2009; Julián et al., 2012). Aunque imprecisa, la FC es la magnitud más fácil de emplear al principio del proceso de aprendizaje, especialmente cuando se trata de datos que se van a comparar únicamente con los del mismo corredor, nunca con los demás, por su carácter subjetivo (Sola y Rodríguez, 2010). En una gráfica el corredor o su compañero anota, cada vez que el corredor pasa por el punto de inicio de su serie, en el eje de abscisas el tiempo transcurrido y en el de ordenadas la FC (Figura 4). Con esta gráfica se puede interpretar fácilmente esta relación entre FC y esfuerzo en las primeras sesiones.

b. La percepción del esfuerzo, empleando como referencia escalas de esfuerzo percibido como la de Borg u otras validadas para escolares como la Children's OMNI Perceived Exertion Scale (Robertson et al., 2001, 2002) o la CERT (Children's Effort Rating Table) (Yelling, Lamb, y Swaine, 2002), entre

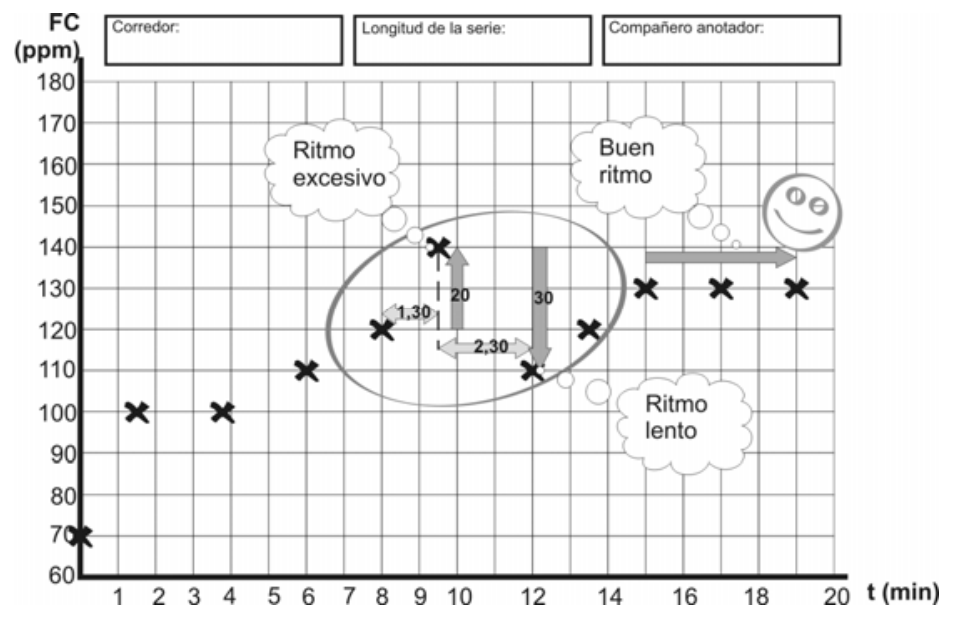

Figura. 4. Gráfica de toma de datos en una sesión de series elaborada por los escolares. Basada en Generelo et al. (2009, p.262) otras, relacionando éstas con las gráficas de FC y tiempos que comentábamos en el apartado anterior. 


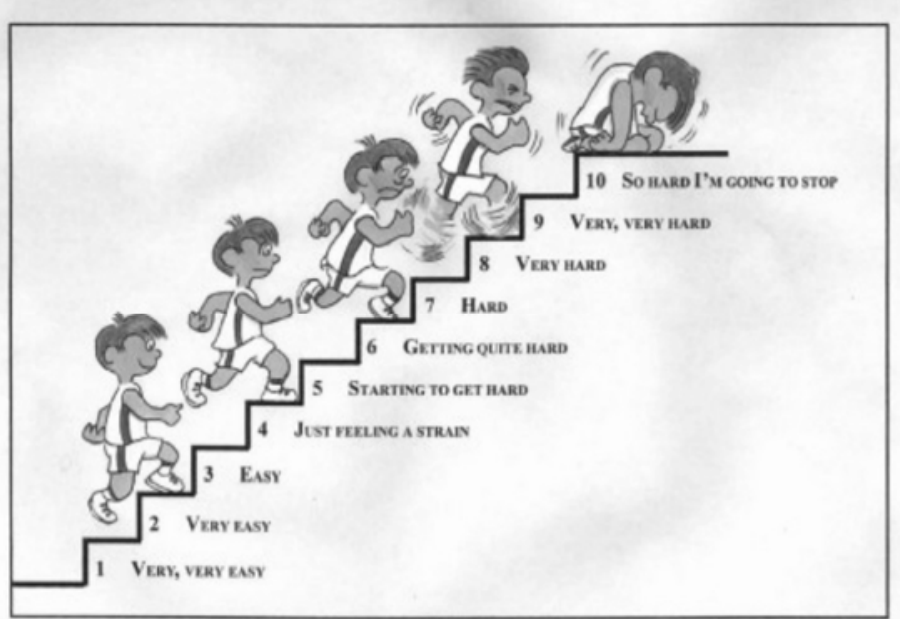

Traducción propia de la escala:

10 Ya es demasiado duro, voy a parar

9 Es muy, muy duro

8 Es muy duro

7 Es Duro

6 Se está haciendo duro de verdad

5 Empieza a ser duro

4 Ya estoy empezando a sentir fatiga

3 Es fácil

2 Es muy fácil

1 Es muy, muy fácil

Figura 5. Índice de clasificación del esfuerzo para escolares o Children’s Effort Rating Table (CERT) (Yelling et al., 2002, p. 161)

c. La importancia de encontrar un ritmo adecuado que les permita completar la distancia o el tiempo propuesto. En este sentido, es importante comprender los conceptos de equilibrio aeróbico o estado estable de lactato (stady state)y el efecto que producen sobre éste los cambios de terreno (Billat, 2002; Billat, Sirvent, Py, Koralsztein y Mercier, 2003). Así mismo, deben comprender el concepto de velocidad crítica (VC) como ritmo de carrera adecuado para mantener el esfuerzo durante largo tiempo (Cárdenas y Mendoza, 2008). Generelo et al. (2009, p.102) apuestan por el empleo del "porcentaje de la velocidad máxima aeróbica” (\%VMA) como magnitud para controlar individualizadamente la intensidad del esfuerzo, siempre que se pueda determinar mediante un test específico (progresivo o continuo) como la Course Navette u otro similar.Estos test se emplearán sólo para comprender el concepto de ritmo adecuado para una CLD, la gráfica de tiempo y series nos muestra a qué velocidad hemos ido realizando las series (Figura. 4) y si hemos conseguido mantener la intensidad, nunca como medio de valuación o selección. Así mismo, la gráfica de FC y tiempos nos sirven para relacionarlo con el esfuerzo. Este recurso es útil cuando las sesiones se desarrollan en circuito homogéneo, sin necesidad de disponer de pulsómetros. Sin embargo, en la naturaleza o en terrenos variados, el pulsómetro sería nuestro mejor aliado. No es necesario disponer de uno para cada corredor, tan sólo poder rotarlo para que vean la evolución de la FC y comprendan el efecto de los cambios de terreno (Generelo et al., 2005; López, 2004).

- Todas estas actividades de entrenamiento deben realizarse en grupo. La pareja es la mejor unidad de trabajo, aunque también pueden ser tríos. De este modo se facilita el control, se comparten las experiencias y se refuerza el ambiente de colaboración del grupo. Así mismo, es imprescindible dedicar momentos para compartir los resultados antes y después de cada actividad, aprovechando tiempos de recuperación o estableciendo como rutina una asamblea al principio y al final de la sesión (Barba et al., 2007). Este proceso de reflexión compartida no puede ser dirigida o monopolizada por el profesor o entrenador, sino que éste debe guiar a los corredores, ofreciendo libertad suficiente para que interpreten la información y saquen sus propias conclusiones.

- Es recomendable que se planteen eventos o carreras acordes con este modelo. En este sentido, las carreras convencionales no son compatibles con el aprendizaje de la CLD, aunque pueden servir cuando ya se ha interiorizado la importancia de los conceptos descritos anteriormente. Estas carreras se plantean con recorridos de diferentes distancias y desniveles, a elegir por los participantes, acompañados por monitores que procurarán regular el ritmo e 
ir fragmentando los grupos según la velocidad que pueden aguantar los corredores. Siempre son mixtas y no atienden a las edades, sino al reto que cada uno se plantee. Al final de la carrera no habrá clasificaciones comparativas entre ellos, tan sólo la recompensa de haber logrado el reto. Por tanto, la información sobre su resultado la conocerá sólo cada corredor (distancia, tiempo, número de series, etc.) También puede haber un reconocimiento público tras haber finalizado con la entrega de algún obsequio a todos los que hayan logrado su reto personal. Si se quiere fomentar el esfuerzo, el obsequio puede ser proporcional a la distancia recorrida. Este tipo de carreras se pueden organizar de tres modos:

a. Recorridos en progresión creciente. Todos comienzan haciendo el primer recorrido. Quienes lo deseen siguen con el segundo, tras finalizar el primero y descansar. Se sigue así sucesivamente hasta realizar todos los recorridos propuestos, que irán siendo de mayor longitud y desnivel. Es el más adecuado cuando los participantes no están habituados a correr, puesto que les ayuda a regular el reto progresivamente (Figura 6).

b. Recorridos diferenciados abiertos. Se plantean diferentes recorridos que permitan "atajar" para regresar con los grupos que vean que no podrán continuar. El monitor acompañante es clave para gestionar esta decisión. Este "atajo" se puede hacer en diferentes puntos, para permitir la nivelación del reto. En ocasiones se regresa por el mismo trayecto de ida. En esta opción, se puede entregar un distintivo a los corredores que van alcanzando hitos más le-

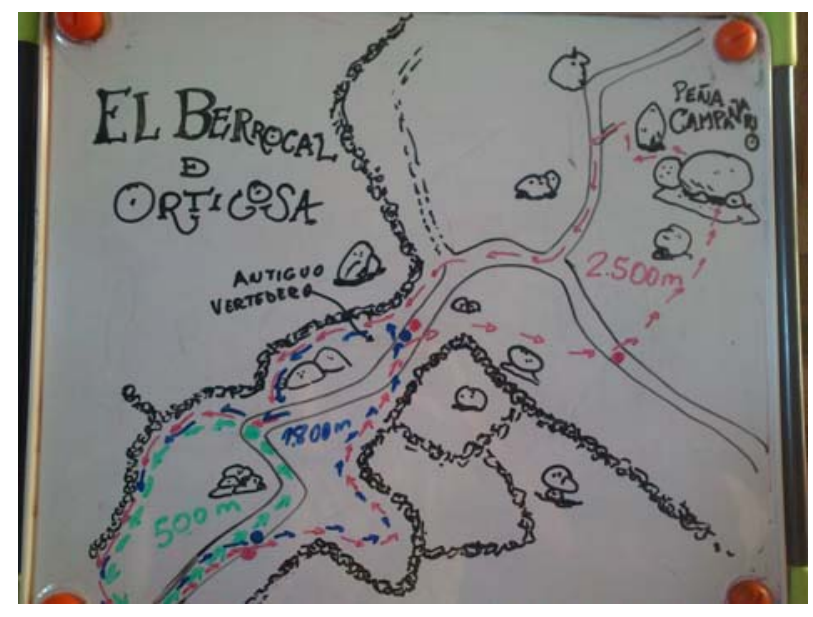

Figura 6. Pizarra con los tres recorridos propuestos en una carrera familiar por el monte (2013). janos, como una lana o pulsera, de tal modo que se motiven por aumentar la distancia. En ningún caso habrá un registro público o clasificación de quiénes han alcanzado cada hito, aunque las comparaciones tras la carrera son a veces inevitables.

c. Recorridos diferenciados cerrados. En este caso los participantes deben saber elegir bien su reto, pues no se puede acortar ni regresar. En todo caso, hay diferentes ritmos en cada recorrido.

\section{UN HORIZONTE EN LAS MONTAÑAS}

Este trabajo es sólo una pieza más de un ambicioso triple objetivo: a) promover el aprendizaje de los deportes en la naturaleza entre los más jóvenes, b) reforzar la presencia de estos deportes en el currículum de Educación Física, justificada por su valor para la salud y la ocupación del tiempo libre y c) fomentar que las entidades responsables de la gestión deportiva (federaciones, clubes, administraciones o empresas) vean el modelo deportivo participativo con mejores ojos y no se centren prioritariamente en el modelo competitivo de rendimiento.

Compartir nuestro trabajo tiene como finalidad promover el trabajo en este sentido y crear colaboraciones futuras que mejoren este modelo y amplíen su repercusión en la práctica deportiva infantil. 


\section{8.- REFERENCIAS}

Barba Martín, J.J., et al. (2007). Aprender a correr con autonomía como aprendizaje relevante en Educación Física. Experiencias en primaria y secundaria. EFDeportes, 105 (Recuperado de: http://www.efdeportes.com/efd105/aprender-a-correr-como-aprendizaje-relevante-en-educacionfisica.htm)

Barba Martín, J.J. y López Pastor, V.M. (coord.) (2006). Aprendiendo a correr a ritmo constante y sostenible en esfuerzos de larga duración. Unidades didácticas y experiencias en Educación Primaria, Secundaria Obligatoria y Bachillerato. Miño y Dávila: Buenos Aires.

Billat, V. (2002). Fisiología y metodología del entrenamiento. De la teoría a la práctica. Paidotribo: Barcelona.

Billat,V., Sirvent, P., Koralsztein, J.P., y Mercier, J. (2003). The concept of maximal lactate steady state: A bridge between biochemistry, physiology and sport science. Sport Medicine, 33, 407-426.

Cárdenas, P.A. y Mendoza, D. (2008). Correr sin agotamiento: ¿qué es la velocidad crítica? EFDeportes, 120 (Recuperado de: http://www.efdeportes.com/efd120/correr-sin-agotamiento-que-es-la-velocidadcritica.htm)

Fernández-Balboa, J.M. (2004). La Educación Física desde una perspectiva crítica: de la Pedagogía venenosa y el currículo oculto hacia la dignidad. En V.M. López-Pastor, R. Monjas Aguado, y A. Fraile Aranda (Coords.), Los últimos diez años de Educación Física Escolar (pp. 215-225) Valladolid: Universidad de Valladolid.

Generelo, E., López, J., Zaragoza, J., Julián, J.A., y Rueda, C. (2005). Un maletín de pulsómetros para la educación física escolar. Actas VI Congreso Internacional de Educación Física y Deporte Escolar. FEADEF. Córdoba.

Generelo, E., Julián, J.A., y Zaragoza, J. (coords.) (2009). Tres vueltas al patio. La carrera de larga duración en la escuela. INDE: Barcelona.

Gill, T. (2007). No Fear. Growing up in a risk averse society. Calouste Gulbenkian Foundation: London

Gill, T. (2010). Nothing ventured... Balancing risks and benefits in the outdoors. English Outdoor Council: Nothingam.

Gracia Bailó, F. (2002). La Educación Física en la escuela rural. Tándem. Didáctica de la Educación Física, 9, 48-58.

Julián, J.A., Generelo, E., García, L., Abarca, A., y Zaragoza, J. (2012). Estrategias para fomentar un clima motivacional óptimo en el contenido de carrera de larga duración en la educación física escolar. Tándem. Didáctica de la Educación Física, 40 (54-66).

Larraz, A. (1997). Experiencias y recursos para la Educación Física en la Escuela Rural. En V.M. López y R. Monjas (coords.), Curso de Verano sobre la Educación Física en la Escuela Rural. E.U. Magisterio de Segovia. Segovia.

Larraz, A. et al. (1998). Una propuesta para trabajar la resistencia aeróbica en Primaria. En Actas de las Jornadas Provinciales de EF de Huesca. Balneario de Panticosa (Huesca). CPR de Sabiñánigo

López, J. (2004). El maletín didáctico de pulsómetros en la escuela. En Actas de las Jornadas Provinciales de Educación Física. Huesca.

Pérez Pueyo, A. (1999a). La "otra" condición física: un planteamiento actitudinal. La significatividad de los conceptos. En Actas IV Congreso de Ciencias del Deporte, Educación Física y Recreación. Lérida. INEFC Lleida. (701-711).

Pérez Pueyo, A. (1999b). La significatividad de los procedimientos en la "otra" condición física: un planteamiento actitudinal. En Actas IV Congreso de Ciencias del Deporte, Educación Física y Recreación (pp. 717-732). Lérida. INEFC Lleida.

Pérez Pueyo, A. (2010). El Estilo Actitudinal: Propuesta metodológica para desarrollar Unidades Didácticas en Educación Física. CEP: Madrid.

Roberts, G.C. y Treasure, D.C. (2003). Motivational determinants of achievement of children in sport. Dossier. (pp. 125-138) (Recuperado de: https://www.golf.se/Global/Tavla/Swedish\%20Golf\%20Team/Achievement\%20golf\%20theory\%20fors kning.pdf)

Roberts, G.C. y Treasure, D.C. (Eds.) (2012). Advances in motivation in sport and exercise. Human Kinetics: Champaign, IL. 
Roberts, G.C., Kleiber, D.A., y Duda, J.L. (1981). An Analysis of Motivation in Children's Sport: The Role of Percived Competence in Participation. Journal of Sport Psychology, 3. (206-216) (Recuperado de: http://journals.humankinetics.com/AcuCustom/Sitename/Documents/DocumentItem/8777.pdf)

Robertson, R., Goss, F., Bell, J.A., Dixon, C.B., Gallagher, T., y Lagally, K.M. (2002). Self-regulated cycling using the Children's OMNI Scale of Perceived Exertion. Medicine Science Sports Exercise, 34(7), 1178-1175.

Robertson, R., Goss, F., Boer J., Gallagher, T., Thompkins, K., Buffalino, G., Balaaekran, C., Meckes, J., Pintar, A. y Williams, A (2001). OMNI scale perceived exertion at ventilator break-point in children: response normalized. Medicine Science Sports Exercise, 34(7), 1168-1175.

Sola, J. y Rodríguez, J.A. (2010). Uso de la frecuencia cardiaca como indicador de la intensidad en las clases de educación física. EFDeportes.com, 148. (Recuperado de: http://www.efdeportes.com/efd148/uso-de-lafrecuencia-cardiaca-en-educacion-fisica.htm)

Yelling, M., Lamb, K.L., y Swaine, I.L. (2002). Validity of a Pictorial Percieved Exertion Scale for Effort Estimation and Effort Production During Stepping Exercise in Adolescent Children. European Physical Education Review, 8, 157 (Recuperado de: http://schools.nyc.gov/NR/rdonlyres/0642B5D7-82AE40B1-AAB6-91CE179022D0/116882/PCERT.pdf)

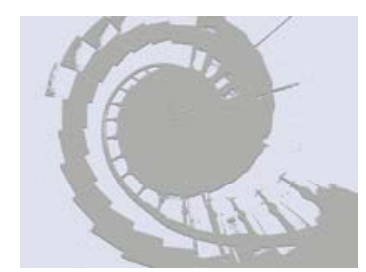

